\title{
ON IRREDUCIBLE MAPS
}

\author{
BY R. BAUTISTA
}

The notion of irreducible map was introduced by M. Auslander and I. Reiten in [3] and plays an important role in the representation theory of artin algebras.

We recall that an artin ring $\Lambda$ is said to be an artin algebra if its center $C$ is an artin ring and $\Lambda$ is finitely generated left $\Lambda$-module. Now choose a complete set $P_{1}, \ldots, P_{s}$ of representatives of the isomorphism classes of indecomposable projectives in $\bmod (\Lambda)$, we will denote by $\operatorname{pr} \Lambda$ the full subcategory of $\bmod \Lambda$ whose objects are $P_{1}, \ldots, P_{s}$. A map $g: X \rightarrow Y$ in $\bmod (\Lambda)$ is said to be irreducible if $g$ is neither a split monomorphism nor a split epimorphism and for any commutative diagram

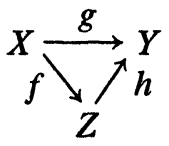

$f$ is a splittable monomorphism or $h$ is a splittable epimorphism.

We study irreducible maps in $\bmod (\Lambda)$ by using properties of the Jacobson radical of $\bmod (\Lambda)$. We recall that the Jacobson radical of $\bmod (\Lambda)$ is the subfunctor $\operatorname{rad}$ of the two variable functor Hom: $(\bmod (\Lambda))^{\mathrm{op}} \times \bmod (\Lambda) \rightarrow \mathrm{Ab}$ defined by

$$
\begin{aligned}
\operatorname{rad}(X, Y) & =\{f \in \operatorname{Hom}(X, Y) \mid 1-g f \text { is invertible for every } g \in \operatorname{Hom}(Y, X)\} \\
& =\{f \in \operatorname{Hom}(X, Y) \mid 1-f h \text { is invertible for any } h \in \operatorname{Hom}(X, Y)\} .
\end{aligned}
$$

It is easy to prove that if $X$ and $Y$ are indecomposables, then $\operatorname{rad}(X, Y)$ consists of all nonisomorphisms, from $X$ to $Y$.

We can prove the following result:

Proposition 1. Let $C$ and $D$ be indecomposables in $\bmod (\Lambda)$. Then

(a) $A$ map $f: C \rightarrow$ is irreducible iff $f \in \operatorname{rad}(C, D)$ and $f \notin \operatorname{rad}^{2}(C, D)$, where $\operatorname{rad}^{2}(C, D)$ consists of all maps of the form $t_{1} t_{2}$ with $t_{2} \in \operatorname{rad}(C, X)$ and $t_{1} \in \operatorname{rad}(X, D)$.

Received by the editors July 9, 1979.

AMS (MOS) subject classifications (1970). Primary 16A64.

(c) 1980 American Mathematical Society $0002-9904 / 80 / 0000-0007 / \$ 02.00$ 
(b) A map

$$
g=\left(\begin{array}{c}
g_{1} \\
\vdots \\
g_{n}
\end{array}\right): C \rightarrow D \amalg \cdots \amalg D
$$

is irreducible iff $\bar{g}_{1}, \ldots, \bar{g}_{n}, \operatorname{rad}(C, D) / \mathrm{rad}^{2}(C, D)$ are linearly independent over $\operatorname{End}(D) / \operatorname{rad} \operatorname{End}(D)$.

Using properties of rad we obtain the result stated below:

THEOREM 1. Suppose $f \in \operatorname{rad}(X, Y)$. Then the following statements are equivalent

(a) $f$ is irreducible,

(b) For any splittable monomorphism $u: C \rightarrow X$ with $C$ indecomposable the composed map fu is irreducible.

(c) For any splittable epimorphism $v: Y \rightarrow D$ with $D$ indecomposable of is irreducible.

If the artin algebra $\Lambda$ is infinite and of finite representation type then we have rather precise information about irreducible maps between indecomposables in $\bmod (\Lambda)$. We are able to prove the following

THEOREM 2. Suppose $\Lambda$ is an infinite artin algebra of finite representation type and let $X$ and $Y$ be indecomposables in $\bmod (\Lambda)$. If we denote by $d$ and $d^{\prime}$ the dimensions of $\operatorname{Hom}(X, Y) / \mathrm{rad}^{2}(X, Y)$ over $\operatorname{End}(X) / \operatorname{rad} \operatorname{End}(X)$ and over End $(Y) / \mathrm{rad} \operatorname{End}(Y)$ respectively, then $d d^{\prime} \leqslant 3$.

We also get information about the middle term of any almost split sequence in $\bmod (\Lambda)$. We recall that the short exact sequence $0 \rightarrow A \stackrel{u}{\rightarrow} B \stackrel{V}{\rightarrow} C \rightarrow 0$ is said to be almost split if (a) the sequence does not split. (b) For any $h: X$ $\rightarrow C$ nonsplittable epi there exists $g$ with $v g=h$. (c) For any $h^{\prime}: A \rightarrow Y$ nonsplittable mono there exists $g^{\prime}: B \rightarrow Y$ with $g u^{\prime}=h^{\prime}$.

THEOREM 3. Assume $\Lambda$ is an infinite artin algebra of finite representation type. Let

$$
0 \rightarrow A \rightarrow n_{1} B_{1} \amalg n_{2} B_{2} \amalg \cdots \amalg n_{s} B_{s} \rightarrow A^{\prime} \rightarrow 0
$$

be an almost split sequence in $\bmod (\Lambda)$ with $B_{i}$ indecomposable, $B_{i} \neq B_{j}$ if $i \neq$ $j$ and $n_{i} B_{i}$ means the direct sum of $n_{i}$ copies of $B_{i}$.

(a) $n_{i} \leqslant 3$ for every $i=1, \ldots, s$.

(b) If for some i $n_{i} \geqslant 2$, then $n_{j}=1$ if $j \neq i$.

(c) If $\Lambda$ is a finite dimensional algebra over an algebraically closed field $k$, then $n_{i}=1$ for any $i$. 
The idea of the proof is the following:

We can assume $\Lambda$ indecomposable, denote by $C$ the center of $\Lambda$. Then $K=$ $C / \operatorname{rad} C$ is a field. Now consider $X$ and $Y$ in $\bmod (\Lambda)$. We define the set $I(X, Y)$ $=\left\{\bar{f} \in \operatorname{rad}(X, Y) / \operatorname{rad}^{2}(X, Y) \mid f\right.$ is an irreducible map $\}$. Now we put $K_{X}^{*}=$ units of $\operatorname{End}(X) / \operatorname{rad} \operatorname{End}(X)$, and the same for $K_{Y}$. In some cases $I(X, Y)$ is an affine $K$-variety and the $K$-algebraic group $K_{X}^{*} \times K_{Y}^{*}$ acts on $I(X, Y)$. Then using properties of irreducible maps [4] and the Gabriel-Tits argument [5] we get our theorem.

Following M. Auslander a skelletally small preadditive category $C$ is said to be prevariety if: (a) Any object in $C$ can be decomposed as finite sum of indecomposable objects in $C$. (b) Any idempotent in $C$ splits. (c) $\operatorname{End}(M)$ for any indecomposable object of $C$ is a local ring. We recall that the Auslander graph $A(C)$ of $C$ is defined as follows:

Choose a complete set of representatives $M_{i}, i \in I$, of all the isomorphism classes of indecomposable objects in $C$. Then the vertices of $A(C)$ are the elements of $I$. We put an arrow from $i$ to $j$ iff there exists an irreducible map $f$ : $M_{i} \rightarrow M_{j}$.

Now we define the Auslander species of $\mathcal{C}$ by attaching to each point $i \in$ $\mathrm{A}(\mathrm{C})$ the division $\operatorname{ring} K_{i}=\operatorname{End}\left(M_{i}\right) / \operatorname{rad} \operatorname{End}\left(M_{i}\right)$, and to each arrow $i \rightarrow j$ in A(C) the $K_{i}-K_{j}$ bimodule $M_{i j}=\operatorname{rad}\left(M_{i}, M_{j}\right) / \operatorname{rad}^{2}\left(M_{i}, M_{j}\right)$.

We note that when $\Lambda$ is an artin algebra and $C=\operatorname{pr}(\Lambda)$ then the Auslander species of $\mathcal{C}$ is just the Dlab-Ringel species of $\Lambda$ (see [6]). As in [7] we can associate to the Auslander species of $\bmod (\Lambda)$ a tensor category $T_{\Lambda}$.

We define $\operatorname{rad}^{i}(X, Y)$ in similar way as $\operatorname{rad}^{2}(X, Y)$ was defined. We put $\operatorname{rad}^{\infty}(X, Y)=\bigcap_{i \geqslant 1} \operatorname{rad}^{i}(X, Y)$. Here $\operatorname{rad}^{\infty}(X, Y)$ is an ideal in $\bmod (\Lambda)$. Then as in [7] we have the following:

Proposition 2. If $\Lambda$ is either an hereditary artin algebra of finite representation type or a finite dimensional algebra over an algebraically closed field $k$, then there exists a full functor $G: T \rightarrow \bmod (\Lambda) / \mathrm{rad}^{\infty}$ such that for any indecomposable module $M$ in $\bmod (\Lambda)$ there exists $M^{\prime}$ in $T_{\Lambda}$ with $G(M) \cong M^{\prime}$.

Observe that if $\Lambda$ is of finite representation type than $\operatorname{rad}^{\infty}(X, Y)=0$ for any $X$ and $Y$ in $\bmod (\Lambda)$.

Using properties of hereditary artin algebras proved in [2] we can describe Ker $G$ in terms of almost split sequences.

\section{REFERENCES}

1. M. Auslander, Representation theory of Artin algebras. I, Comm. Algebra 1 (1974), 177-268.

2. M. Auslander and M. I. Platzeck, Representation theory of hereditary Artin algebras, Representation Theory of Algebras, (Proc. Conf. Temple University, Philadelphia, 1976, pp. 369-424). Lecture Notes in Pure and Applied Math., vol. 37, Dekker, New York, 1978.

3. M. Auslander and I. Reiten, Representation theory of Artin Algebras. III, Almost split sequences, Comm. Algebra 3 (1975), 239-294. 
4. - Representation theory of Artin algebras. IV, Invariants given by almost split sequences, Comm. Algebra 5 (1977), 443-518.

5. I. N. Bernstein, I. M. Gelfand and V. A. Ponomariev, Coxeter functors and Gabriel's theorem, Uspeshi Mat. Nauk 28 (1973), 19-33.

6. V. Dlab and C. M. Ringel, Indecomposable representation of graphs and algebras, Mem. Amer. Math. Soc. 173 (1976).

7. - On algebras of finite representation type, J. Algebra 33 (1975), 306-394.

INSTITUTO DE MATEMÁTICAS UNIVERSIDAD NACIONAL AUTÓNOMA DE MÉXICO, AREA DE LA INVESTIGACIÓN CIENTÍFICA, CIRCUITO EXTERIOR, C. U. MEXICO 20, D. F. MÉXICO 